\title{
Total and Segmental Colon Transit Time Study in Functional Constipation: Comparison With Healthy Subjects
}

\author{
Prasad A. Bhate, ${ }^{a}$, Jatin A. Patel ${ }^{\mathrm{a}}$, Pathik Parikh ${ }^{\mathrm{a}}$, Meghraj A. Ingle ${ }^{\mathrm{a}}$, Anniruddha Phadke ${ }^{\mathrm{a}}$, \\ Prabha D. Sawant ${ }^{\mathrm{a}}$
}

\begin{abstract}
Background: Constipation is a common problem worldwide. Constipation can be primary or secondary. Primary constipation is subdivided in slow transit constipation, normal transit constipation, and dyssynergic defecation. Colon transit time (CTT) is the most basic and primary tool in evaluating disorders of colonic motility. CTT helps to differentiate between types of constipation and plan the treatment.
\end{abstract}

Methods: Fifty functional constipation patients and 25 healthy controls were asked to ingest four gelatin capsules (each containing five radio-opaque markers) at 0,12 and 24 hours. An abdominal X-ray was taken at 36 hours. Total or segmental CTT was measured after calculating the number of markers remaining in each segment at 36 hours on abdominal X-ray.

Results: Mean CTT in healthy controls in our study was 15.4 hours which is shorter than western population. Total CTT is significantly higher in constipation group ( 23 hours) compared to healthy subjects (15.4 hours). Transit time in right segment was significantly high in constipation group than healthy population (14.2 vs. 8.3 hours). Total as well as segmental transit times are slightly higher in females as compared to males in both the groups, however not statistically significant. To the best of our knowledge, there are no studies from India that compared the CTTs in functional constipation and healthy controls.

Conclusion: Radio-opaque marker study for CTT is a simple and reliable technique for evaluation of constipation. Patients with functional constipation have significantly longer CTT than healthy population. Total CTT is much less in this study population compared to west. There is need to establish standards for slow colon transit.

Keywords: Functional constipation; Segmental colon transit time; Total colon transit time

Manuscript accepted for publication February 16, 2015

aDepartment of Gastroenterology, Lokmanya Tilak Municipal Medical College and Hospital, Sion, Mumbai, India

${ }^{\mathrm{b}}$ Corresponding Author: Prasad Ashok Bhate, Department of Gastroenterology, Lokmanya Tilak Municipal Medical College and Hospital, Sion, Mumbai, India.Email: prasadbhate07@gmail.com

doi: http://dx.doi.org/10.14740/gr642w

\section{Introduction}

Constipation is a common problem worldwide [1]. Constipation can be primary or secondary. Primary constipation is subdivided into slow transit, normal transit constipation and dyssynergic defecation. Though in most patients constipation is caused by irritable bowel syndrome (IBS) in the West, in about half of these patients, it is caused either by slow transit constipation (STC) or fecal evacuation disorders (FEDs) [1]. There are various methods for investigating constipation like plain abdominal radiograph, contrast enema, defecating proctogram, measurement of colonic transit time and anorectal manometry [1].

Out of the above tests, measurement of colon transit time (CTT) is the most basic and primary tool in evaluating disorders of colonic motility. CTT helps to differentiate between types of constipation and plan the treatment. Various methods are available for measuring CTT. The standard technique is radio-opaque marker test which is a most widely used method because it is easy to perform and cost effective. Only disadvantage of this method is radiation exposure. Radionuclide scintigraphy and wireless motility capsules are other techniques used to measure CTT [2].

In a country like India, it is not easy to investigate the patient of constipation because of lack of widespread availability of anorectal manometry and barium defecography. So CTT becomes an important test for the management of patients with constipation.

CTT test protocol in western countries is use of 20 radioopaque markers to be given at 0,24 and $48 \mathrm{~h}$ each, followed by an abdominal X-ray at $72 \mathrm{~h} \mathrm{[3-5].} \mathrm{However,} \mathrm{Indians} \mathrm{have} \mathrm{rapid}$ gut transit, so above protocol may not be suitable for measuring CTT. To overcome this problem, some investigators have attempted to decrease the interval between ingestion of markers and abdominal X-ray. Nabar et al developed a protocol in which 20 radio-opaque markers were given at 0,9 and $18 \mathrm{~h}$

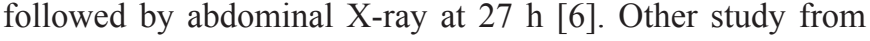
India used a protocol of administering 20 radio-opaque markers at 0,12 and $24 \mathrm{~h}$ each followed by an abdominal X-ray at 12,24 and $36 \mathrm{~h}$ [7]. Recently Ghoshal et al reported a different protocol in which radio-opaque markers were given at 0,12 and $24 \mathrm{~h}$ followed by X-ray taken at 36 and $60 \mathrm{~h} \mathrm{[8] \text {. }}$

There is paucity of CTT studies from India in patients of functional constipation. 
Table 1. Basic Characteristics

\begin{tabular}{lll}
\hline & Functional constipation & Healthy subjects \\
\hline Age (SD) & 33.3 years $(11.8)$ & 32.5 years $(10.3)$ \\
Gender (M:F) & $26: 24$ & $12: 13$ \\
BMI (SD) & $22 \mathrm{~kg} / \mathrm{m}^{2}(1.3)$ & $21.2 \mathrm{~kg} / \mathrm{m}^{2}(1.7)$ \\
\hline
\end{tabular}

\section{Methods}

\section{Subjects}

Patients, diagnosed as functional constipation (Rome 3 criteria), attending GI OPD of a large tertiary public hospital were included in study. Diagnostic work-up, including colonoscopy, barium enema and/or anorectal manometry as well as blood tests to rule out hypothyroidism, hypercalcemia was performed. Patients with abnormal findings suggestive of an organic disease in any of the investigations were excluded. Other exclusion criteria were pregnancy, severe medical illness, alcoholism, patients taking drugs affecting motility and history of major abdominal surgery. Controls were healthy subjects without any gastrointestinal complaints.

\section{Measurement of CTT}

CTT was measured by using radio-opaque markers. Patients were asked to ingest four gelatin capsules (each containing five radio-opaque markers) at 0,12 and $24 \mathrm{~h}$. Abdominal X-ray was taken at $36 \mathrm{~h}$ from the time of ingestion of first dose of markers. Localization of markers on X-ray was done by identifying body structures as described in a study by Arhan et al [9]. Markers located on right of the vertebral spinous processes above a line from the fifth vertebra to the right pelvic outlet were assigned to the right colon. Markers to the left of the vertebral spinous processes and above an imaginary line from the fifth lumbar vertebra to the anterior superior iliac crest were assigned to the left colon. And markers inferior to a line from the pelvic brim on the right and the superior iliac crest on the left were judged to be in the right sigmoid colon and rectum [10].

Total or segmental CTT was measured after calculating the number of markers remaining in each segment at $36 \mathrm{~h}$ on abdominal X-ray [11].

CTT (or segmental transit time) $=12 / 20 \times(n)$ hours, where $\mathrm{n}$ is the sum of the markers on the X-ray film (or in the delineated segments).

Subjects were asked to follow their usual daily routine and diet, to avoid unusual intensive physical activity and alcohol intake.

\section{Statistical analysis}

Unpaired Student's $t$-test was used to compare the mean transit time between the two groups. All patients completed the informed consent form. The study protocol was approved by the ethics committee of our institute.

\section{Results}

Fifty functional constipation patients and age and sex matched 25 healthy controls were enrolled (Tables 1-3). The duration of constipation ranged between 8 months to 20 years in FC patients.

\section{Discussion}

Radio-opaque marker test is a simple and convenient method to measure CTT. There is a paucity of literature regarding CTT study in functional constipation patients in India. In the present study, multiple capsule and single film technique was used to ensure patient compliance and minimize radiation exposure [10]. As gut transit is faster in India, it is recommended to shorten the duration between marker ingestion and X-ray abdomen [11].

Mean CTT in healthy controls in our study was $15.4 \mathrm{~h}$ which is shorter than western population $[12,13]$. Our findings are consistent with other studies from India $[6,8,12]$. This is probably attributed to higher fiber content in Indian diet. The average stool frequency in India is one per day [14]. In western literature, the transit times in right, left and rectosigmoid colon are similar [10]. In our study, the transit time in right segment is higher as compared to left and rectosigmoid region.

We compared the CTTs in patients with functional constipation and healthy controls. We found that total CTT is significantly higher in constipation group $(23 \mathrm{~h})$ compared to healthy subjects $(15.4 \mathrm{~h})$. Transit time in right segment was significantly high in constipation group than healthy population (14.2 vs. $8.3 \mathrm{~h}$ ). The difference of left segment and rectosigmoid transit time between both groups was not significant.

Table 2. Mean Transit Time in Hours

\begin{tabular}{llll}
\hline & Functional constipation & Healthy subjects & \\
\hline $\begin{array}{l}\text { Total colon transit time (h) } \\
\text { Segmental transit time (h) }\end{array}$ & 23 & 15.4 & $\mathrm{P}<0.05$ \\
$\quad$ & & & \\
Right & 14.2 & 8.3 & $\mathrm{P}<0.05$ \\
Left & 6.5 & 4 & n.s. \\
Rectosigmoid & 5 & 3.5 & n.s. \\
\hline
\end{tabular}


Table 3. Mean Transit Time in Male and Female

\begin{tabular}{lllllll} 
& \multicolumn{2}{c}{ Functional constipation } & & \multicolumn{2}{c}{ Healthy subjects } & \\
\cline { 2 - 3 } & Male & Female & & Male & Female & \\
\hline Total colon transit time (h) & 21 & 25 & & 14.2 & 17 & n.s. \\
Segmental transit time (h) & & & & & \\
$\quad$ Right & 12.9 & 15 & 7.8 & 8.5 & n.s. \\
Left & 5.8 & 6.8 & 3.7 & 4.6 & n.s. \\
Rectosigmoid & 4.7 & 5.9 & 3 & 4 & n.s. \\
\hline
\end{tabular}

Gender comparison of transit times shows that total as well as segmental transit times are slightly higher in females as compared to males in both the groups, however not statistically significant. One study suggested that left segmental transit time was higher in females [15] and suggested progesterone may be responsible for prolongation of transit time in females.

To the best of our knowledge, there are no studies from India that compared the CTTs in functional constipation and healthy controls. So our study provides an important finding that patients with functional constipation have longer total and right segmental transit time in comparison to healthy controls. Limitation of our study is that normal range of transit time in healthy population in India is not established. Also the colonic transit is affected by age as well as gender. Rarely multiple markers may stick to one another and produce difficulty in reading the film.

In conclusion, radio-opaque marker study for CTT is a simple and reliable technique for evaluation of constipation. Patients with functional constipation have significantly longer CTT than healthy population. Total CTT is much less in this study population compared to west. There is need to establish standards for slow colon transit.

\section{Conflicts of Interest}

No conflicts of interest to be declared.

\section{References}

1. Locke GR, 3rd, Pemberton JH, Phillips SF. AGA technical review on constipation. American Gastroenterological Association. Gastroenterology. 2000;119(6):1766-1778.

2. Wald A. Motility disorders of the colon and rectum. Curr Opin Gastroenterol. 2012;28(1):52-56.

3. Rao SS, Ozturk R, Laine L. Clinical utility of diagnostic tests for constipation in adults: a systematic review. Am J Gastroenterol. 2005;100(7):1605-1615.
4. Berman IR, Manning DH, Harris MS. Streamlining the management of defecation disorders. Dis Colon Rectum. 1990;33(9):778-785.

5. Bouchoucha M, Devroede G, Arhan P, Strom B, Weber $\mathrm{J}$, Cugnenc PH, Denis P, et al. What is the meaning of colorectal transit time measurement? Dis Colon Rectum. 1992;35(8):773-782.

6. Nabar AA, Bhatia SJ, Abraham P, Ravi P, Mistry FP. Total and segmental colonic transit time in non ulcer dyspepsia. Indian J Gastroenterol. 1995;14(4):131-133.

7. Pai CG, Kurian G. A modified radiographic method for estimating segmental colonic transit time in subjects with rapid gut transit. Indian J Med Res. 1999;110:22-26.

8. Ghoshal UC, Gupta D, Kumar A, Misra A. Colonic transit study by radio-opaque markers to investigate constipation: validation of a new protocol for a population with rapid gut transit. Natl Med J India. 2007;20(5):225-229.

9. Arhan P, Devroede G, Jehannin B, Lanza M, Faverdin C, Dornic C, Persoz B, et al. Segmental colonic transit time. Dis Colon Rectum. 1981;24(8):625-629.

10. Metcalf AM, Phillips SF, Zinsmeister AR, MacCarty RL, Beart RW, Wolff BG. Simplified assessment of segmental colonic transit. Gastroenterology. 1987;92(1):40-47.

11. Chaussade S, Roche H, Khyari A, Couturier D, Guerre J. [Measurement of colonic transit time: description and validation of a new method]. Gastroenterol Clin Biol. 1986;10(5):385-389.

12. Jayanthi V, Chacko A, Gani IK, Mathan VI. Intestinal transit in healthy southern Indian subjects and in patients with tropical sprue. Gut. 1989;30(1):35-38.

13. Licht HM. Irritable bowel syndrome. Definitive diagnostic criteria help focus symptomatic treatment. Postgrad Med. 2000;107(3):203-207; quiz 277.

14. Thankappan KR, Narendranathan M. Normal Bowel Habits: Results of a study in Kerala. Indian Journal of Gastroenterology. 1992;11(suppl 1):A3.

15. Jung HK, Kim DY, Moon IH. Effects of gender and menstrual cycle on colonic transit time in healthy subjects. Korean J Intern Med. 2003;18(3):181-186. 